\title{
Conditions for the existence of stable strange quark matter
}

\author{
N.A. Dondi ${ }^{1}$,, A. Drago ${ }^{1}$, and G. Pagliara ${ }^{1}$ \\ ${ }^{1}$ Dip. di Fisica e Scienze della Terra dell'Università di Ferrara and INFN Sez. di Ferrara, Via Saragat 1, I- \\ 44100 Ferrara, Italy
}

\begin{abstract}
We discuss the possible existence of absolutely stable strange quark matter within three different types of chiral models. We will show that confinement plays a crucial role in determining the conditions for the Bodmer-Witten hypothesis to hold true. We discuss also which are the phenomenological signatures, related to measurements of masses and radii of compact stars, which would prove the existence of strange quark stars.
\end{abstract}

\section{Introduction}

The so called Bodmer-Witten hypothesis on the absolute stability of strange quark matter $($ see $[1,2]$ and [3] for a review) still represents an open issue of nuclear and particle physics and its validity would have numerous important phenomenological implications in astrophysics (strange quark stars and strangelets in cosmic rays) and, possibly, also in cosmology (strangelets as a component of baryonic dark matter). Verifying this hypothesis in terrestrial experiments is however very difficult because of the large net strangeness carried by this form of strongly interacting matter. On the other hand, theoretical calculations of the mass and density of strange quark matter droplets and of the thermodynamic properties of bulk strange quark matter are affected by large uncertainties due to the intrinsic difficulty of solving QCD at finite (but not asymptotically large) baryon densities. In this short contribution we will analyze two aspects related to the possibility of the existence of strange quark matter. First, we will show that this hypothesis can be fulfilled also in a class of quark models which feature not only confinement (as the MIT bag model) but also chiral symmetry. Specifically, we will show results obtained within the so-called chiral chromodielectric model [4].

The second aspect concerns the astrophysical measurements of masses and radii of compact stars. Precise radii measurements (which will be feasible in the near future) could indeed unambiguously check the validity of the Bodmer-Witten hypothesis. Let us define $R_{1.4}$ as the radius of a $1.4 M_{\odot}$ compact star. As found in $[5,6]$ it is not possible to have a unique family of compact stars satisfying at the same time the condition $R_{1.4} \lesssim 11 \mathrm{~km}$ and $M_{\max } \leq 2 M_{\odot}$. This conclusion has been confirmed also by other recent analyses [7]. If future measurements will confirm the existence of stars for which $R_{1.4} \lesssim 11 \mathrm{~km}$ [8], then one can conclude that strange quark stars co-exist with hadronic stars, as proposed in Refs.[9-11] In this two families scenario compact and light stars are hadronic stars whereas large and massive stars are strange quark stars.

a e-mail: nicolaandrea.dondi@student.unife.it 


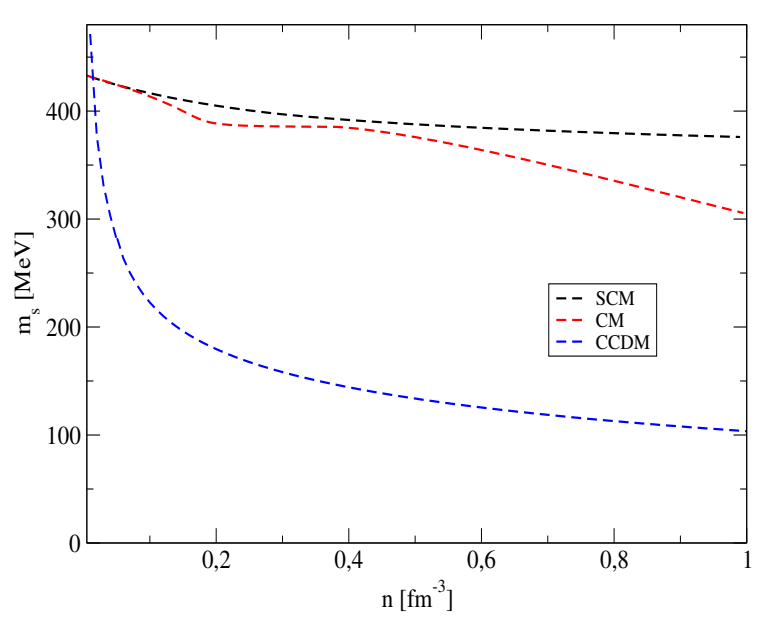

Figure 1. Baryon density dependence of the strange quark mass in the three chiral models considered in this work.

Since the $\sim 2 M_{\odot}$ compact stars are, in this scenario, interpreted as strange quark stars that implies that the density of bound strange quark matter (i.e. the density corresponding to the minimum of the energy per baryon) is between 1-2 times nuclear matter saturation density. It is important to remark that although the absolute minimum of the energy per baryon of nuclear matter is located at a density similar to the density of strange quark matter they differ concerning the strangeness content. This implies that a direct transition from the metastable nucleonic state to the ground state of quark matter is suppressed because it requires multiple weak decays [2].

\section{Quark matter equation of state}

It is a fact that ab-initio QCD calculations in the regime of high baryon density are still unfeasible and therefore to describe the matter of compact stars one has to rely on quark/hadronic models which capture at least some of the features of QCD. When exploring the conditions for the Bodmer-Witten hypothesis to hold true, the MIT bag model (which implements in a simple way QCD confinement) turns out to have a significant freedom in the choice of parameters to allow for the existence of stable strange quark matter, see the seminal paper [12]. In particular, the Bodmer-Witten hypothesis is satisfied when small values of the strange quark current mass are adopted. On the other hand, in chiral models, such as the NJL model [13] this possibility seems to be ruled out, see also the recent work of Ref. [14]. Notice that while the MIT bag model, in its simplest version, features confinement but not chiral symmetry, the opposite is true in (simple) chiral models. It is therefore clear that the BodmerWitten hypothesis could be fulfilled if a subtle interplay between confinement and spontaneous chiral symmetry breaking exists. We consider here different kinds of SU(3) chiral models in which quarks interact through meson exchange. What turns out to be the most important microscopic quantity for the validity of the Bodmer-Witten hypothesis is the density dependence of the strange quark mass which in turn is determined by the quark-meson couplings. To obtain a minimum of the energy per baryon lower than the one of nuclear matter (in order for strange quark matter to be absolutely 


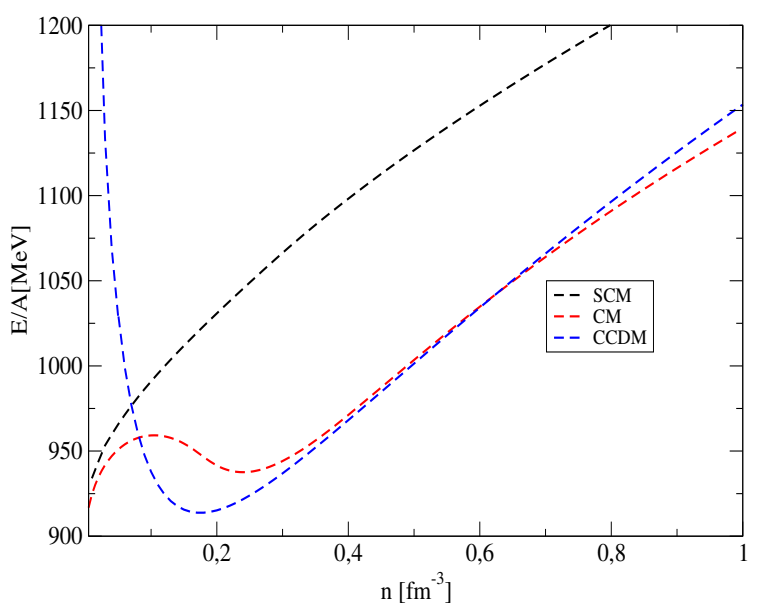

Figure 2. Energy per baryon as a function of the baryon density for the three chiral models considered in this work.

stable), it is necessary that the strange quark mass decreases significantly at densities close to nuclear saturation density.

We analyze the density dependence of the strange quark mass by using three different chiral models for beta-stable and charge neutral quark matter within the Hartree approximation:

- The basic version of the SU(3) Chiral Model (CM) which describes deconfined quarks interacting through scalar and vector mesons.

- The Scaled Chiral Model (SCM) which additionally incorporates the anomalous breaking of the scale invariance through the action of the dilaton scalar field.

- The Chiral Chromodielectric model (CCDM) which allows to simulate quark confinement through the action of the color dielectric field.

Among the three models, only the CCDM features dynamical confinement. Indeed in this model the effect of the dielectric field is to make the quark masses divergent in vacuum.

We show in Fig. 1 and Fig. 2 the strange quark mass and the energy per baryon for typical sets of parameters used in these models (see details in a forthcoming paper [15]). The parameter fixing is performed, as in [16], by reproducing the tree level mass spectrum of pseudoscalar mesons. From Fig. 1 one can notice that the strange quark mass decreases very slowly with density within the CM and SCM while it presents a significant drop, within the CCDM, already at a density comparable to nuclear saturation density. Concerning the minimum of the energy per baryon, it is not possible to find a parameter set allowing for the stability of strange quark matter for CM and SCM (in agreement with previous findings $[14,17])$; in Fig. 2 we show only one typical example for each model: within the CM a minimum of the energy per baryon could be found but its value is larger than the one of nuclear matter. Within the SCM, no minimum of the energy per baryon is found unless in some specific fine tuned sets of parameters. ${ }^{1}$. On the other hand, within the CCDM, it is possible to find

\footnotetext{
${ }^{1}$ Both in CM and SCM it is possible to find minima of the energy per baryon smaller than the one of nuclear matter but without a net strangeness fraction. Moreover, chiral symmetry restoration in the up and down quark sectors occurs at too low densities. Such cases are clearly ruled out.
} 


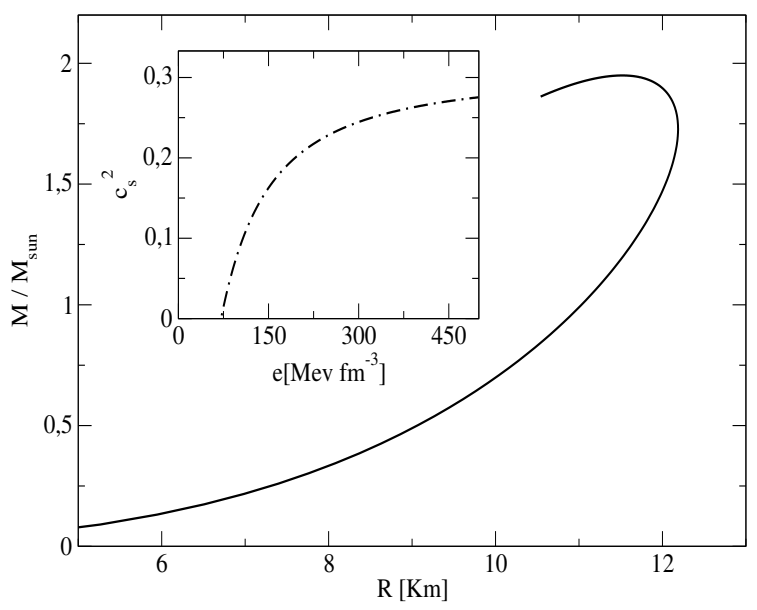

Figure 3. Mass-Radius relation corresponding to the CCDM equation of state. In the insert, we display the speed of sound as a function of the energy density.

sets of parameters for which the Bodmer-Witten hypothesis is fulfilled. In the case shown in Fig.2, the minimum of the energy per baryon is $E / A \sim 913 \mathrm{MeV}$ and it is realized at a density slightly larger than the nuclear saturation density. This result is explained by the fast drop of the strange quark mass, see Fig.1, which in turn is related to a density dependence of the masses that is introduced by the confinement dynamics. An important constraint on quark matter equation of state stems from the maximum mass of compact stars. To reach values of maximum masses close to $2 M_{\odot}$, one must introduce within the CCDM also vector meson contributions. We use here a quark-vector meson interaction term analogous to the quark-scalar one, namely:

$$
\mathcal{L}_{i n t}=-\frac{\sqrt{2} g_{\sigma}}{\chi}(\bar{q} M q)-\frac{\sqrt{2} g_{\omega}}{\chi}\left(q^{\dagger} V_{0} q\right)
$$

where $M, V_{0}$ are respectively the scalar and vector meson matrices in the mean field approximation. To our knowledge, no attempt to introduce the vector mesons in the CCDM is present in the literature. The quark star mass-radius relation as well as the speed of sound in dense quark matter, obtained within the CCDM model, are displayed in Fig.3. The maximum mass is of $\sim 1.94 M_{\odot}$ and thus compatible (within error bars) with the astrophysical measurements $[18,19]$. We expect that a calculation that introduces also the Fock term in the field equations would provide larger maximum masses. Finally, we would like to remark that the results obtained within the CCDM model and here presented correspond to a choice of the parameter set which is compatible with parameter sets used in the literature for studying hadronic physics observables, see [20] and Refs. therein.

\section{Maximum mass and radii of strange quark stars}

We discuss now which are the astrophysical observations that could test the Bodmer-Witten hypothesis. For the sake of simplicity we use here a parametrization of the quark matter equation of state in which the speed of sound $c_{s}$ is constant. The relation between pressure and energy density is thus 


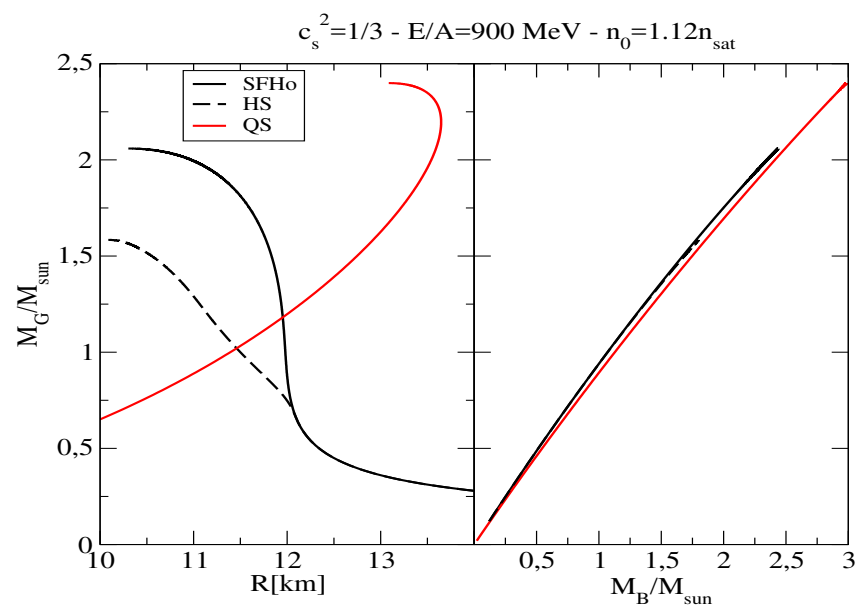

Figure 4. (Left panel): mass radius plot for for nucleonic stars, hadronic stars (as obtained by using the SFHo equation of state [25]) and strange quark stars (as obtained by using a constant-speed equation of state with parameters tuned in order to achieve a maximum mass of $2.4 M_{\odot}$ ). In the right panel, the corresponding relations between baryonic mass and gravitational mass are displayed.

given by $p=c_{s}^{2}\left(e-e_{0}\right)$ where $e_{0}$ represents the energy density at zero pressure (i.e. the energy density of a droplet of quark matter). We note that this equation of state has been also used in Refs.[21] to investigate maximally compact stellar configurations and in Ref.[6] to construct hybrid stars. By indicating with $n_{0}$ the baryon density corresponding to $e_{0}$ one can determine the following relation between pressure and $n$ by simply using the thermodynamic relation $p=n^{2} \frac{\partial / n}{\partial n}$ :

$$
p=k\left(\left(n / n_{0}\right)^{1+c_{s}^{2}}-1\right)
$$

where $k=\frac{e_{0} c_{s}^{2}}{1+c_{s}^{2}}$, see also [22]. Different sets of the three parameters $c_{s}, e_{0}$ and $n_{0}$ determine different possible equations of state with the corresponding mass-radius curves. In many calculations of the quark matter equation of state it results that $c_{s}^{2} \lesssim 1 / 3$ (see [6, 23]) and we will therefore keep it fixed to $1 / 3$. The values of $n_{0}$ and $e_{0}$ are on the other hand completely unknown. A few constraints can however be used: $830 \mathrm{MeV} \lesssim e_{0} / n_{0}=E / A<930 \mathrm{MeV}$ if strange quark matter is assumed to be absolutely stable (the Bodmer-Witten hypothesis) while preserving the stability of Iron with respect to two-flavor quark matter [2]. Moreover, we impose that $n_{0} \gtrsim n_{\text {sat }}$ i.e. the baryon density of strange quark matter droplets must not be smaller than the nuclear matter saturation density. This constraint is satisfied both in the MIT bag model and the CCDM once the parameters are fixed to reproduce basic properties of hadrons such as the radii [20].

In Fig.4, left panel, we show an example of quark star mass-radius curve obtained by requiring that the maximum mass is of $2.4 M_{\odot}$ (which is the value inferred for the black widow pulsar [24]) and that $e_{0} / n_{0}=900$, i.e. only $30 \mathrm{MeV}$ smaller than the binding energy of nuclear matter. The maximum mass constraint allows to fix $n_{0}=1.12 n_{\text {sat }}$. Notice that it is possible to achieve such high values of the maximum mass in quark models provided that $n_{0}$ is close to nuclear saturation density. For comparison we display also the mass-radius relation for the nucleonic equation of state SFHo [25] without (solid line) and with hyperons and deltas (dashed line), [26]. It is remarkable that while quark stars (with masses above $1 M_{\odot}$ ) have radii larger than nucleonic and hadronic stars, they are more 
bound than them: by looking at the relation between baryonic mass and gravitational mass (right panel of Fig.4) one notices that at fixed baryonic mass strange quark stars are lighter than nucleonic and hadronic stars, see also Ref.[9]. It is clear therefore that a process of conversion of a hadronic star into a quark star is energetically convenient even if the radius of the final configuration is larger than the radius of the initial configuration. Notice that the gravitational mass of a strange star is smaller than the one of a hadronic star (with the same baryonic mass) just because the Witten hypothesis is satisfied, almost independently from the parameters' values. The scenario of the conversion of hadronic stars into strange quark stars has been developed in several papers, see Refs. [9-11, 27, 28] and the phenomenological connections with gamma-ray-bursts have been studied in Refs. [29, 30]. It is important to stress that in this scenario the two families of stars, hadronic stars and quark stars, do coexist and that the transition between the two branches is provided by the appearance, in a hadronic star, of hyperons: once a sizable fraction of strangeness is present through hyperons, the (metastable) stellar system can convert into a pure strange quark star. The appearance of hyperons, as obtained by many calculations, should take place around 2-3 times saturation density, which in turn implies that only hadronic stars more massive than about $1.5-1.6 M_{\odot}$ can convert, see $[10,11]$.

Presently, the highest measured mass is the one of PSR J0348+0432, $M=2.01 \pm 0.04 M_{\odot}$ [19], but a candidate with a higher mass does exist: the black widow pulsar with an estimated mass of $2.4 M_{\odot}$ [24]. Moreover, the observations of internal plateaux in short gamma-ray-bursts originating from the merger of two neutron stars seem also to suggest the existence of stars with masses significantly larger than $2 M_{\odot}[31,32]$. Strange quark stars could have maximum masses larger than $2 M_{\odot}$ (see Fig.4-5) provided that the baryon density of strange quark matter droplets is close enough to the density of saturation of nuclei, see also [21]. This can be seen in Fig.5 in which we display the lines of constant maximum mass in the plane of the two parameters $n_{0} / n_{\text {sat }}$ and $E / A$. Notice that, on the other hand, it is very difficult to obtain hadronic stars with masses of $2 M_{\odot}$ or above because of the softening of the equation of state related to the appearance of hyperons and deltas. In this respect, measurements of masses larger than $2 M_{\odot}$ would favor the strange quark star scenario. We remark that this scenario is not in contradiction with neutron matter calculations and terrestrial laboratory experiments. Indeed the conversion of metastable hadronic stars could be triggered only when the central density of such stars is high enough that a significant amount of strangeness appears.

Let us now discuss radii measurements. Future experiments, such as NICER, will measure the radius of a few compact stars with an error of about $1 \mathrm{~km}$. As discusses in [10] and as one can see in Fig.4, it is clear that if $R_{1.4} \lesssim 11 \mathrm{~km}$ the scenario of the two families of compact stars, and therefore the existence of strange quark stars, will be proven ${ }^{2}$.

\section{Conclusions}

We have discussed the possibility of the existence of strange quark matter first within a purely theoretical approach, by using three different chiral models for quark matter and after within phenomenological approach through the measurements of masses and radii of compact stars. The Bodmer-Witten hypothesis could be fulfilled only if spontaneous chiral symmetry breaking and dynamical confinement are both implemented in the adopted quark model. In particular we have shown that a pure SU(3) quark meson chiral model, even in the presence of the additional dilaton field, does not allow for the existence of strange quark matter. Instead, in a simple confining chiral model, the chiral chromo-dielectric model, it is possible to find a window of parameters for which strange quark matter

\footnotetext{
${ }^{2}$ Notice that the model we have adopted for the nucleonic equation of state, the SFHo model, predicts a radius $R_{1.4}$ of about $12 \mathrm{~km}$. This value is compatible with ab-initio calculations of neutron matter as obtained within the chiral effective field theory approach and quantum Monte-Carlo simulations [33].
} 


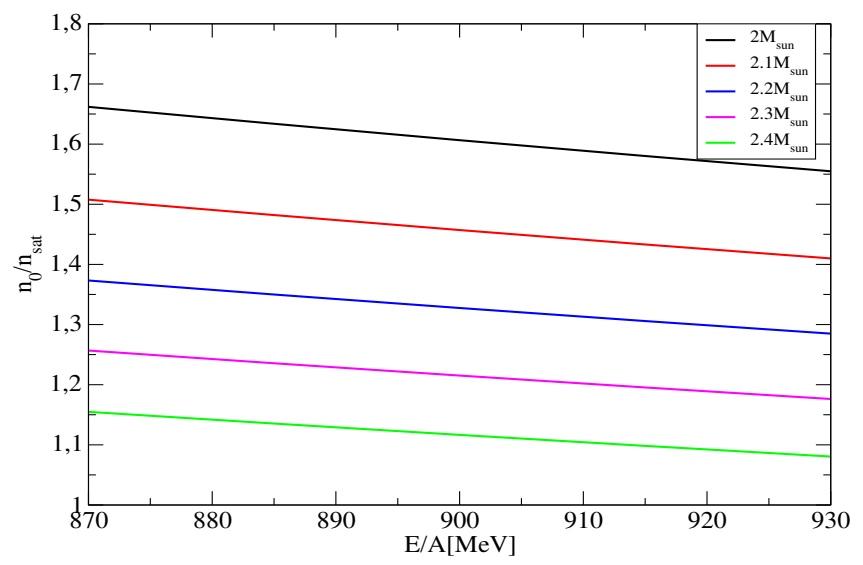

Figure 5. Lines of constant maximum mass as function of the parameters $E / A$ and $n_{0} / n_{\text {sat }}$.

is absolutely stable. It is remarkable that the sets of parameters corresponding to these cases, are quite similar to the sets of parameters usually adopted for the study of hadronic observables.

Finally, we have discussed what are the implications for the equation of state from the existence of massive compact stars and how future measurements of radii can be used to test fundamental properties of dense matter. In particular, a strong indication of the validity of the Bodmer-Witten hypothesis will be measurements of $R_{1.4} \lesssim 11 \mathrm{~km}$. The existence of stars as massive as $2 M_{\odot}$ (or more) would indicate that if the Bodmer-Witten hypothesis is correct, the (second) minimum of the energy per baryon of strongly interacting matter is located at a density slightly above the nuclear matter saturation density. Differently from the minimum corresponding to nuclear matter, this second minimum would contain a net strangeness fraction close to $1 / 3$. The possibility to search for this kind of matter also in terrestrial experiments would be in this case very promising.

\section{References}

[1] A. R. Bodmer, Phys. Rev. D 4 (1971) 1601. doi:10.1103/PhysRevD.4.1601

[2] E. Witten, Phys. Rev. D 30 (1984) 272.

[3] F. Weber, Prog. Part. Nucl. Phys. 54 (2005) 193.

[4] H. J. Pirner, Prog. Part. Nucl. Phys. 29 (1992) 33.

[5] A. Kurkela, E. S. Fraga, J. Schaffner-Bielich and A. Vuorinen, Astrophys. J. 789 (2014) 127.

[6] M. G. Alford, G. F. Burgio, S. Han, G. Taranto and D. Zappalà, Phys. Rev. D 92 (2015) no.8, 083002.

[7] J. Lattimer, talk given at the workshop "Nuclear Physics, Compact stars and Compact star Merger 2016" (NPCSM 2016), Kyoto 2016.

[8] F. Ozel, D. Psaltis, T. Guver, G. Baym, C. Heinke and S. Guillot, Astrophys. J. 820 (2016) no.1, 28.

[9] A. Drago, A. Lavagno and G. Pagliara, Phys. Rev. D 89 (2014) no.4, 043014. 
[10] A. Drago, A. Lavagno, G. Pagliara and D. Pigato, Eur. Phys. J. A 52 (2016) no.2, 40.

[11] A. Drago and G. Pagliara, Eur. Phys. J. A 52 (2016) no.2, 41.

[12] E. Farhi and R. L. Jaffe, Phys. Rev. D 30 (1984) 2379.

[13] M. Buballa and M. Oertel, Phys. Lett. B 457 (1999) 261.

[14] T. Klahn and T. Fischer, Astrophys. J. 810 (2015) no.2, 134.

[15] N. A. Dondi, A. Drago, A.Lavagno, G. Pagliara in preparation.

[16] A. Zacchi, R. Stiele, J. Schaffner-Bielich. Phys. Rev. D 92.4 (2014) , 045022.

[17] P. Wang et Al. Phys. Rev. C 67.1 (2003), 015210.

[18] P. Demorest, T. Pennucci, S. Ransom, M. Roberts and J. Hessels, Nature 467 (2010) 1081.

[19] J. Antoniadis et al., Science 340 (2013) 6131.

[20] A. Drago, U. Tambini and M. Hjorth-Jensen, Phys. Lett. B 380 (1996) 13.

[21] J. M. Lattimer and M. Prakash, arXiv:1012.3208.

[22] J. L. Zdunik, Astron. Astrophys. 359 (2000) 311.

[23] P. Bedaque and A. W. Steiner, Phys. Rev. Lett. 114 (2015) no.3, 031103.

[24] M. H. van Kerkwijk, R. Breton and S. R. Kulkarni, Astrophys. J. 728 (2011) 95.

[25] A. W. Steiner, M. Hempel and T. Fischer, Astrophys. J. 774 (2013) 17.

[26] A. Drago, A. Lavagno, G. Pagliara and D. Pigato, Phys. Rev. C 90 (2014) no.6, 065809.

[27] A. Drago and G. Pagliara, Phys. Rev. C 92 (2015) no.4, 045801.

[28] G. Pagliara, M. Herzog and F. K. Röpke, Phys. Rev. D 87 (2013) no.10, 103007.

[29] A. Drago, A. Lavagno, B. Metzger and G. Pagliara, Phys. Rev. D 93 (2016) no.10, 103001.

[30] A. G. Pili, N. Bucciantini, A. Drago, G. Pagliara and L. Del Zanna, MNRAS 462 (2016) L26.

[31] H. J. Lü, B. Zhang, W. H. Lei, Y. Li and P. D. Lasky, Astrophys. J. 805 (2015) no.2, 89.

[32] A. Li, B. Zhang, N. B. Zhang, H. Gao, B. Qi and T. Liu, Phys. Rev. D 94 (2016) 083010.

[33] J. M. Lattimer and M. Prakash, Phys. Rept. 621 (2016) 127. 